\title{
ESTUDIO CUANTITATIVO SOBRE EL USO DOCENTE DE HERRAMIENTAS TELEFORMATIVAS EN EL ÁMBITO DE LA PROGRAMACIÓN Y BASES DE DATOS
}

\author{
Pablo César Muñoz Carril; pmunoz@udc.es \\ Mercedes González Sanmamed; mercedes@udc.es \\ Universidad de A Coruña
}

\section{RESUMEN}

El artículo que presentamos muestra los resultados más significativos obtenidos en un estudio cuantitativo tipo "survey", desarrollado con docentes de la Universidad de A Coruña que utilizaban sistemas teleformativos como suplemento a sus clases presenciales.

Uno de los objetivos clave de dicha investigación se centraba en conocer cuál era el nivel de aplicación y uso que el profesorado universitario realizaba respecto a herramientas teleeducativas pertenecientes al área de programación y bases de datos.

Los análisis inferenciales realizados determinaron que existían variables de tipo profesional (como la "experiencia docente utilizando entornos virtuales" y la "categoría administrativa") que influían de forma significativa en el nivel de aplicación y uso que mostraba el profesorado en el área de programación y bases de datos bajo contextos teleformativos.

PALABRAS CLAVE: Profesorado universitario, programación, bases de datos, e-learning, educación superior.

\section{ABSTRACT}

This article shows most relevant results obtained in a survey developed with professors from the University of A who use e-learning systems to complement their lecture classes.

The main objective of the research was to identify how much the professors used telematic applications and tools in the programming area and databases at the e-learning environments.

The inferential analysis performed showed that there are certain variables (such as "teaching experience using virtual environments" and "administrative category"), which significantly influenced the level of application and use that showed teachers in the programming area and databases at the e-learning environments. 
KEYWORDS: Faculty, programming, databases, e-learning, higher education.

\section{1.- INTRODUCCIÓN}

No cabe duda de que las universidades españolas, conscientes de las ventajas inherentes de los sistemas de teleformación, han ido implantado secuencialmente entornos virtuales de enseñanza-aprendizaje con el fin de actuar como recurso complementario a las acciones formativas impartidas "cara a cara" (véanse los estudios de Area, 2001; Barro, 2004, 2006; Cabero, 2002; Infante, 2004; Prendes, 2009), posibilitando de esta forma la puesta en marcha de sistemas de enseñanza cada vez más flexibles.

Sin embargo, en este vertiginoso contexto de cambio vehiculizado a través de las TIC, la aplicación de acciones de e-learning bajo la concepción de la enseñanza flexible, abre diversos frentes de cambio o renovación a considerar desde el ámbito de la tecnología educativa. Estos frentes de cambio son, según Salinas (1999):

- Cambios en las concepciones: cómo funciona el aula, definición de los procesos didácticos, identidad del docente, etc...

- Cambios en los recursos básicos,

- contenidos (materiales,....) esto es, digitalización y rediseño de los contenidos de los seminarios de los cursos.

○ infraestructuras (acceso a las redes, alumnos a Internet,....).

○ uso abierto (manipulables por el profesor, por el alumno...)

- eficacia didáctica (coste/beneficio,...)

- Cambios en las prácticas

- de los profesores (de transmisor de conocimientos a facilitador de aprendizaje)

- de los estudiantes (transformar de escuchadores pasivos a activos gestores de su propio proyecto de autoformación).

Partiendo de la tipología señalada en líneas precedentes, los resultados de investigación que mostraremos en el presente artículo se circunscriben en el ámbito de la práctica docente, ya que el uso de sistemas de teleformación implica la asunción de nuevos conocimientos, funciones, tareas y competencias por parte del profesorado (Adell y Sales, 1999; Llorente, 2006; Marcelo, 2006; Muñoz Carril y González Sanmamed, 2009). En particular, nos centraremos en conocer cuál es el nivel de uso de aquellas competencias tecnopedagógicas desarrolladas por el profesorado de primer, segundo y tercer ciclo de la Universidad de A Coruña que utiliza sistemas de e-learning en su práctica docente.

\section{2.- DESCRIPCIÓN DEL PROCEDIMIENTO Y METODOLOGÍA UTILIZADA.}

En un contexto amplio, entendemos por metodología aquella que se refiere a "un diseño por medio del cual el investigador selecciona procedimientos de recogida y análisis de los datos para investigar un problema específico" (McMillan y Schumacher, 2005, p. 12). Esto implica que la metodología de una investigación es sistemática e intencional, puesto que los 
procedimientos no son actividades casuales, sino que están planificados para obtener datos sobre el problema de investigación planteado.

Evidentemente la metodología utilizada, va a estar en clara dependencia del tipo de enfoque empleado por el investigador: cuantitativo o cualitativo ${ }^{1}$. En nuestro caso, una vez valoradas las potencialidades, condicionamientos y limitaciones que supone adoptar uno $u$ otro enfoque, nos hemos decantado finalmente por realizar un estudio cuya modalidad de investigación es de carácter cuantitativo.

Es preciso destacar que, a su vez, la modalidad de investigación (cuantitativa, como ya hemos mencionado) informa sobre el diseño de exploración más apropiado, entendiendo por diseño de investigación aquel que "describe los procedimientos para guiar el estudio, incluyendo cuándo, de quién y bajo qué condiciones serán obtenidos los datos. En otras palabras, el diseño indica cómo se prepara la investigación, qué le pasa a los sujetos y qué métodos de recogida se utilizan" (McMillan y Schumacher, 2005, p. 39)

En este sentido, bajo la modalidad de investigación cuantitativa se incluyen, a su vez, dos grandes tipos de diseño: el experimental y el no experimental. Nuestra investigación se posiciona bajo el ámbito de la modalidad no experimental de tipo "encuesta".

En lo que concierne al esquema del diseño de investigación, resulta evidente que éste se muestra condicionado en gran medida por el tipo de metodología adoptada. Por ello, acorde con nuestros presupuestos teóricos y metodológicos y en un esfuerzo de síntesis, hemos tratado de perfilar en la figura 1 los sucesivos momentos que configuraron, en líneas generales, el proceso de investigación.

Presentamos por tanto, un modelo organizativo del proceso en el que están representados aquellos aspectos que consideramos necesarios en las distintas fases que se han ido configurando en nuestro estudio. No es nuestro propósito presentar una relación exhaustiva de todos los pasos que conviene realizar para dar cuenta de la multiplicidad de elementos implicados, sino simplemente, ofrecer un marco en el que se reflejen nuestras diversas preocupaciones y los diferentes factores que a nuestro parecer procede atender.

Conviene destacar además, que el esquema de diseño de investigación (figura 1) está basado y realizado a partir de las recomendaciones de diversos expertos como Cohen y Manion (1990), Bisquerra (2004), McMillan y Schumacher (2005) y Buendía, Colás y Hernández (1998).

\footnotetext{
${ }^{1}$ Huelga destacar que hoy en día cada vez se hace más presente la pluralidad y complementariedad metodológica (Torrado, 2004, p. 231).
} 


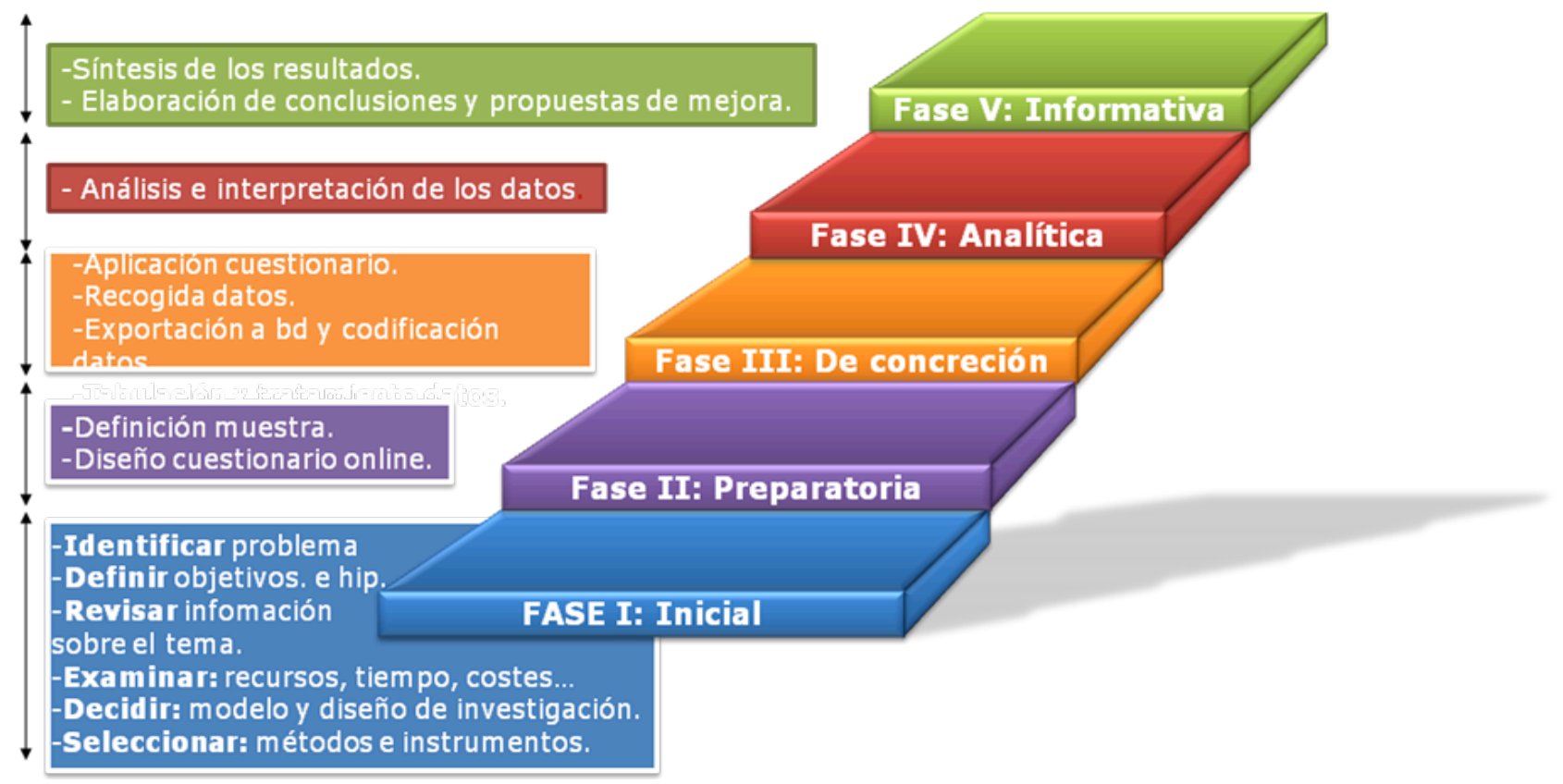

Figura 1.- Fases del diseño de la investigación.

\section{1.- Población y muestra objeto de estudio.}

A efectos de nuestro estudio, la población queda definida por el profesorado de la Universidade da Coruña de primer, segundo y tercer ciclo que impartió docencia a través del sistema teleformativo de dicha institución, denominado "Facultad Virtual", durante el curso académico 2006-2007.

La imposibilidad de que todo el profesorado pudiese participar en la investigación, nos llevó a seleccionar una muestra representativa de la población objeto de estudio, de modo que la representatividad de dicha muestra nos permitiese poder generalizar los datos obtenidos a la población.

Es preciso destacar que a la hora de seleccionar la muestra, hemos seguido las recomendaciones establecidas por Sabariego (2004, p. 144) y Manzano y Braña (2005, pp. 9598), de forma que se han garantizado las dos condiciones básicas que afectan a la selección de la muestra: la representatividad y el tamaño.

Respecto a la representatividad, hemos conseguido que la muestra sea un fiel reflejo de las características principales del conjunto de la población. Tal y como se puede observar en la tabla 1 se ha obtenido un porcentaje muestral representativo según ámbitos científicos.

Además, la participación del profesorado en el estudio en función de su agrupación por áreas de conocimiento, ha sido elevada, quedando registradas un total de 73 áreas de conocimiento.

Por otra parte, de los cuarenta y cuatro departamentos existentes en la Universidade da Coruña aparecen representados un total de cuarenta y dos, a excepción del departamento "galegoportugués, francés y lingüística" y el de "Tecnología y ciencia de la representación gráfica"; lo cual sin duda alguna constituye una muestra representativa a nivel departamental. 


\begin{tabular}{|l|r|r|r|r|}
\hline \multirow{2}{*}{ Ámbito científico } & \multicolumn{2}{|c|}{ Población } & \multicolumn{2}{c|}{ Muestra } \\
\cline { 2 - 5 } & Frecuencia & Porcentaje & Frecuencia & Porcentaje \\
\hline CIENTÍFICO-SANITARIO & 154 & $24,52 \%$ & 35 & $21,1 \%$ \\
\hline HUMANIDADES & 48 & $7,64 \%$ & 10 & $6,0 \%$ \\
\hline TECNOLÓGICO & 245 & $39,01 \%$ & 69 & $41,6 \%$ \\
\hline JURÍDICO-SOCIAL & 181 & $\mathbf{2 8 , 8 2 \%}$ & 52 & $31,3 \%$ \\
\hline TOTALES & $\mathbf{6 2 8}$ & $\mathbf{1 0 0 \%}$ & $\mathbf{1 6 6}$ & $\mathbf{1 0 0 , 0 \%}$ \\
\hline
\end{tabular}

Tabla 1.- Distribución de la población y de la muestra por ámbitos científicos ${ }^{2}$.

En función de la edad también existe representatividad en las diferentes categorías que hemos prefijado. En concreto, existe una participación de un 19,3\% de profesores con edades comprendidas entre 25 y 35 años, un $46,4 \%$ con edades entre 36 y 45 años, un 23,5\% de docentes con edades situadas en el intervalo de 46 a 55, y finalmente un 10,2\% de sujetos de entre 56 a 65 años.

Según la experiencia docente universitaria utilizando entornos virtuales también se han obtenido resultados representativos y equilibrados. En concreto, han participado un $15,7 \%$ de profesores con menos de un año de experiencia utilizando sistemas de e-learning, un 22,3\% con una experiencia entre 1 y 2 años, un 33,1\% entre 3 y 4 años, un 10,2\% entre 5 y 6 años, y un $15,1 \%$ poseen más de 6 años de experiencia.

Por categoría administrativa también se ha obtenido una muestra representativa, habiendo participado catedráticos universitarios $(7,2 \%)$, titulares de universidad $(30,7 \%)$, catedráticos de Escuela Universitaria (6,6\%), titulares de Escuela Universitaria (18,7\%), profesores ayudantes $(4,2 \%)$, profesores asociados $(13,3 \%)$, contratados doctores $(7,8 \%)$, contratados laborales interinos $(1,2 \%)$ y profesores colaboradores $(9 \%)$.

En lo que atañe a la determinación del tamaño muestral y dado que partimos de una población finita constituida por un total de 628 sujetos, podemos calcular cuál es el número mínimo de profesores necesarios que deben formar parte del estudio para que éste sea significativo.

Así pues y para determinar el tamaño muestral, partimos de la formulación para poblaciones finitas propuesta por Arnal, del Rincón y Latorre (1992):

\footnotetext{
${ }^{2}$ Es preciso Indicar que los datos poblacionales de los que partimos han sido facilitados por la unidad de teleformación de la Universidade da Coruña (previa autorización de la Vicerrectoría de Calidad y Harmonización Europea).

Además es necesario recalcar que los 628 profesores y profesoras que forman la población son docentes que realmente han utilizado durante el curso 2006-2007 la Facultad Virtual y por tanto han interactuado en mayor o menor medida con su alumnado a través de esta plataforma de e-learning. El criterio utilizado para poder determinar qué profesores realmente usaron la FV, fue contabilizar si sus materias estaban disponibles para el alumnado en formato virtual. De esta forma, gracias a los datos oficiales facilitados por la unidad de teleformación de la UDC pudimos discriminar entre aquellos profesores que tenían acceso a la FV (lo cual no quería decir que la utilizasen), de aquellos otros que virtualizaron sus materias dándolas de alta en el curso 2006/07, de forma que el alumnado pudiese acceder a las mismas.
} 


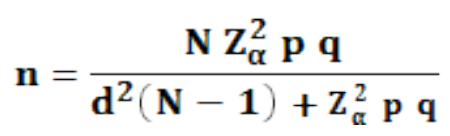

$\mathrm{N}=$ Total de la población

$\mathbf{Z}_{\alpha}^{2}=1,962$ (seguridad del 95\%)

$\mathrm{p}=$ proporción esperada (en este caso $5 \%=0.05$ )

$q=1-p$ (en este caso $1-0.05=0.95)$

$\mathrm{d}=$ precisión (en este caso deseamos un 3\%)

La sustitución de la fórmula anterior por sus valores proporcionó el tamaño siguiente para la muestra de nuestra investigación:

$$
\mathrm{n}=\frac{628 *(1.96)^{2} * 0.05 * 0.95}{(0.03)^{2} *(628-1)+(1.96)^{2} * 0.05 * 0.95}=153.45 \cong 154
$$

Se recibieron 178 respuestas, que tras ser tabuladas y filtradas a fin de evitar distorsiones en los resultados finales quedaron en 166, por lo que podemos afirmar que la muestra con la que se ha trabajado es ligeramente superior a la requerida.

Una vez realizadas estas precisiones acerca de la representatividad y tamaño de la muestra, resulta de notoria obligatoriedad que nos detengamos un instante en comentar la técnica de muestreo utilizada. En un primer momento optamos por realizar un muestreo aleatorio simple, porque de este modo todas las unidades que componen la población (628 profesores en nuestro caso) tienen la misma probabilidad de pertenecer a la muestra, circunstancia que hace catalogar a ésta de "muestra autoponderada" (Manzano y Braña, 2005, p.100). No obstante, llevar a cabo esta técnica implicaba una serie de condicionamientos básicos, como por ejemplo disponer de una base de datos con nombres, apellidos y direcciones de correo electrónico del profesorado, a partir de la cual:

- Poder asignar un número a cada individuo de la población.

- Realizar la selección de los sujetos que formarán parte de la muestra de forma aleatoria (utilizando tablas de números aleatorios o subprogramas de números aleatorios de programas informáticos).

- Enviar al correo electrónico del profesorado que resultó elegido la dirección web donde teníamos hospedado el cuestionario on-line.

Si bien el proceso parece sencillo, nos encontramos con trabas a nivel legal, puesto que desde la unidad de teleformación de la UDC, dependiente del Vicerrectorado de Calidad y Harmonización Europea, no nos podían facilitar, como es lógico, los datos de la población objeto de estudio. Este hecho nos condicionó a tener que acogernos a otro tipo de técnica de muestreo. En concreto, y gracias a la ayuda brindada por parte del vicerrector de Calidad y Harmonización Europea de la UDC, decidimos utilizar una técnica de muestreo no probabilística denominada accidental, consistente en recurrir a los informantes en base a su disponibilidad o facilidad de acceso (Solanas, 1997). Este tipo de técnica también recibe el nombre de muestreo casual (Arnal, del Rincón y Latorre, 1992; Bisquerra, 2004) o muestreo por conveniencia (Cohen y Manion, 1990; McMillan y Schumacher, 2005). Somos conscientes de que a través de esta 
técnica de muestreo debemos ser cautelosos a la hora de generalizar los resultados obtenidos, puesto que estarán restringidos a las características de la muestra. No obstante, como ha quedado constancia en líneas anteriores, se ha conseguido reunir a una muestra con unas características representativas del conjunto de la población.

En definitiva, el proceso seguido fue dar a conocer a toda la población objeto de estudio la investigación que estábamos desarrollando, de forma que aquellos profesores que voluntariamente quisiesen participar en el estudio tan sólo debían conectarse a una dirección web en la que contestar al cuestionario on-line.

Para conseguir la máxima participación de nuestro público objetivo se optó por publicitar el estudio de dos formas:

- En primer lugar realizamos una carta de presentación en donde se explicitaban los objetivos de nuestra investigación y se invitaba al profesorado de la UDC (que utilizaba entornos virtuales como complemento a sus clases presenciales), a participar en la investigación. Dicha carta fue enviada por correo electrónico a la secretaría del Vicerrectorado de Calidad y Harmonización Europea ${ }^{3}$ de la UDC. A su vez, desde este vicerrectorado, y previo permiso y consentimiento del vicerrector, se envió la carta a la población objeto de estudio.

- Desde la unidad de teleformación de la UDC, dependiente del VCHE, se incluyo en el apartado "noticias" de la plataforma de e-learning de esta universidad, un hipervínculo hacia la dirección web donde estaba albergado el cuestionario on-line. De esta forma, aquellos docentes que accediesen con su clave a la "Facultad Virtual", verían en primera instancia la propuesta que le hacíamos para que participasen en el estudio.

A tenor del alto porcentaje de profesores que participaron en la investigación podemos decir que ambas medidas tuvieron éxito.

\section{2.- El instrumento de recogida de datos: el cuestionario on-line.}

La consideración de nuestro objeto de estudio, la pretensión de conseguir información en un período de tiempo concreto (curso académico 2006-2007) y el amplio número de sujetos participantes, aconsejaba la utilización de la técnica del cuestionario para la recogida de datos. Además, a estas razones debemos unirle otras como el hecho de que el cuestionario es considerado como una técnica de indagación respetable y válida, que bien construida y aplicada, puede ser una estrategia muy apropiada para la obtención de datos, permitiendo recoger información cuantificable y determinada previamente por los evaluadores (Cohen y Manion, 1990).

No obstante, la decisión de optar por utilizar el cuestionario como instrumento de recogida de información, estuvo amparada además por una revisión de las opiniones de los expertos (Best, 1982; Buendía et al., 1998; Cohen y Manion, 1990; Fox, 1981; McMillan y Schumacher, 2005; Torrado, 2004), la cual nos permitió establecer no solamente las posibilidades y ventajas del uso del cuestionario como instrumento de recogida de información, sino también sus posibles limitaciones.

Seguidamente especificamos aquellas virtudes y ventajas inherentes al cuestionario on-line, que hemos identificado como decisivas a la hora de emplear este instrumento para la recogida de datos:

\footnotetext{
${ }^{3}$ A partir de ahora VCHE.
} 
- En primer lugar, el cuestionario nos permite obtener información de un elevado número de personas de forma simultánea y en un período temporal relativamente corto. De hecho, y gracias a la versión on-line creada, pudimos recibir de forma automatizada a través de una base de datos todos los cuestionarios contestados por los docentes.

- Económicamente hablando, tiene unos costes discretos. En nuestro caso particular, y dado que el cuestionario se aplicó a través de Internet, los únicos gastos fueron los relativos a la adquisición de un hosting con soporte en lenguaje PHP y MySQL, para hospedar la base de datos.

- Desde un punto de vista temporal, el profesorado podía contestar al cuestionario en el día, la hora y momento que considerasen oportuno. Este hecho suponía facilidades a la hora de contestar al propio cuestionario. De facto, hubo profesores que contestaron al mismo en días no laborables e inclusive a altas horas de la noche. Paralelamente, hay que considerar que el hecho de que los docentes tuviesen tiempo suficiente para responderlo podía proporcionar respuestas más meditadas que las que obtendríamos por otros métodos de indagación.

- Otra ventaja significativa, es la facilidad y rapidez con la que se pueden codificar, tabular y analizar los datos. En nuestro caso empleamos la versatilidad de software específico como Macromedia DreamWeaver 8 para la creación del cuestionario en HTML y el archivo de procesamiento de datos en lenguaje PHP4; WAMP y Appserv como servidores locales para realizar pruebas en modo localhost; phpMyAdmin como panel de control de la base de datos en lenguaje MySQL; Microsoft Excel como programa de exportación de los datos y SPSS 15 para importar los datos de Excel y realizar los correspondientes análisis estadísticos.

- Además de todos estos beneficios hay que considerar un aspecto, a nuestro juicio fundamental, que es el anonimato y la protección de datos que se asegura a los participantes. El hecho de poder expresar opiniones amparándose en el anonimato podía ser un aspecto que condicionase la participación del profesorado. Además tiene un valor añadido, que no es otro que estimular la sinceridad en las respuestas.

- Por último, es preciso destacar que la aplicación del cuestionario por Internet también lleva implícitas una serie de fortalezas (algunas de las cuales ya hemos comentado en líneas precedentes). Para Torrado (2004, p. 245), éstas se circunscriben en que se evitan errores de codificación, saltos de pregunta, etc., también permite acceder a poblaciones de difícil acceso ${ }^{4}$.

\footnotetext{
${ }^{4}$ De hecho si no utilizásemos un cuestionario aplicado a través de Internet, sería prácticamente imposible conseguir una tasa de respuesta tan alta por parte del profesado de la UDC que utiliza entornos virtuales, ya que el acceso a la población era muy limitado. Además éramos conocedores del hecho de que la práctica totalidad de los docentes poseen conexión a Internet en sus despachos, e inclusive tienen la posibilidad de utilizar la conexión WI-FI en todos los campus de la Universidade da Coruña, por lo que a nivel de recursos e infraestructuras resultaba idónea la aplicación de un cuestionario on-line.
} 
Sin embargo, y a pesar de las ventajas expresadas anteriormente, hemos de ser conscientes de las limitaciones e inconvenientes que presenta el uso de cuestionarios on-line como instrumento de recogida de datos. Siguiendo a González Sanmamed (1993, p. 593), uno de los principales peligros es que el cuestionario es "una técnica de exploración indirecta e impersonal, se corre el peligro de que sólo devuelvan el cuestionario una pequeña parte de los sujetos, pudiendo quedar afectada la muestra e incidiendo negativamente en la representatividad que pretendía".

También hay que tener presente que no siempre es posible tener la certeza de que la información proporcionada por los encuestados se corresponde con la realidad; tanto es así, que las respuestas pueden variar en diferentes períodos temporales, en función de la situación personal del sujeto en el momento en que se aplica el cuestionario, o de otras circunstancias como por ejemplo la situación emocional de la persona (González Sanmamed, 1993).

Además de todo ello, Torrado (2004, p. 245) señala una serie de desventajas específicas para las encuestas realizadas por Internet. De todas ellas, la que realmente podía suponer un aspecto que afectase al estudio eran los riesgos derivados de posibles fallos informáticos y de aquellos relativos al diseño, gestión, mantenimiento y procesamiento de los datos. Para ello tuvimos que realizar, previa aplicación del cuestionario on-line, multitud de pruebas y testeos que asegurasen la fiabilidad de la aplicación.

Cabe indicar que para tratar de evitar en la medida de lo posible estos problemas, en nuestro estudio se han tomado en consideración las observaciones realizadas por un grupo de expertos, tanto en lo que se refiere a la elaboración y diseño del cuestionario, como a la redacción, organización y estructura de las preguntas formuladas a lo largo del mismo, de forma que el cuestionario garantizase unos criterios de validez y fiabilidad.

Nuestro objetivo por tanto, no fue otro que traducir los objetivos planteados en la investigación, por preguntas concretas que recopilasen la realidad que pretendíamos estudiar. De esta forma, el cuestionario cumple en función de los objetivos prefijados una triple función: describir las características, valoraciones y opiniones de la muestra; relacionar las diferentes variables que se incluyen en el instrumento de medición; y finalmente, aunque en menor medida, explicar y predecir determinadas situaciones y hechos.

Centrándonos en el proceso de diseño del cuestionario on-line, establecemos seguidamente las características que lo definen y que se han tenido principalmente en consideración para su construcción.

a) Desde un punto de vista de su formato, los ítems utilizados siguen un formato cerrado (también denominado ítems estructurados, selección de respuesta o cerradofinalizado).

b) En cuanto al tipo de escala valorativa, hemos utilizado en los diferentes bloques de los que consta el cuestionario, una escala tipo Likert de cinco puntos, por tanto impar. De este modo damos la posibilidad a aquellos sujetos indecisos de posicionarse en un término medio.

c) Según su estructura, el cuestionario se articula en un total de 248 ítems divididos en 5 bloques de contenido. El segundo bloque, denominado "competencias técnicas", está dividido a su vez en cinco categorías ("ofimática", "infografía y multimedia", "programación y bases de datos" y "utilidades e-learning"). En este trabajo nos ceñiremos exclusivamente a la categoría referida al nivel de aplicación/uso de 
herramientas del área de programación y bases de datos por parte del profesorado. (ítems 10.12 al 10.15).

d) Por el fin científico u objetivo para el que se ha diseñado, el cuestionario es de tipo descriptivo y explicativo (Torrado, 2004).

e) Por su forma de administración es un cuestionario aplicado por vía electrónica.

f) Según su dimensión temporal, el cuestionario es de tipo seccional, sincrónico o transversal, debido a que la información es recogida una única vez en un período de tiempo limitado (finales del curso académico 2006-2007) y de una población definida (profesorado de la Universidade da Coruña).

g) Según el sentido y momento de recogida de información es una encuesta retrospectiva.

Hay que tener en consideración que el instrumento de medida es una herramienta construida ad-hoc para este trabajo de investigación, en consecuencia, resultó de vital importancia que se cumpliesen dos características psicométricas fundamentales: la fiabilidad y la validez. Para hallar la fiabilidad se utilizó el índice de consistencia interna Alpha de Cronbach, obteniéndose un $\alpha=0,956$, lo que indica una muy buena fiabilidad. En lo que atañe a la validez, se contó con la colaboración de reputados expertos ${ }^{5}$ de diferentes universidades españolas que actuaron en calidad de jueces expertos para valorar la validez de contenido del instrumento. Las valoraciones realizadas por el grupo de expertos nos sirvieron para tomar decisiones a la hora de poder rechazar, mejorar o mantener en el cuestionario cada uno de los ítems.

Una vez elaborado el cuestionario en su versión impresa, se procedió a su diseño digital. En la siguiente figura 2 se representa un esquema completo con las diversas fases desarrolladas.

Finalmente, antes de aplicar el cuestionario, se realizó una prueba piloto seleccionando a un grupo de profesores de la UDC a través de un muestreo intencional. De esta forma conseguiríamos varios objetivos:

- Saber el tiempo que lleva completar el cuestionario.

- Conocer las opiniones de los encuestados, obteniendo una crítica constructiva acerca de la estructura del cuestionario.

- Valorar, a través de las ideas aportadas por los encuestados, la idoneidad de suprimir, rediseñar o mejorar determinados ítems.

- Determinar si en el momento de cubrir el cuestionario tuvieron algún problema de índole técnica.

\footnotetext{
${ }^{5}$ Se contó con la colaboración de tres profesores universitarios del área de métodos de investigación (dos catedráticos y una doctora), y dos profesores universitarios especializados profesionalmente en elearning y en tecnología educativa (un catedrático y un doctor).
} 


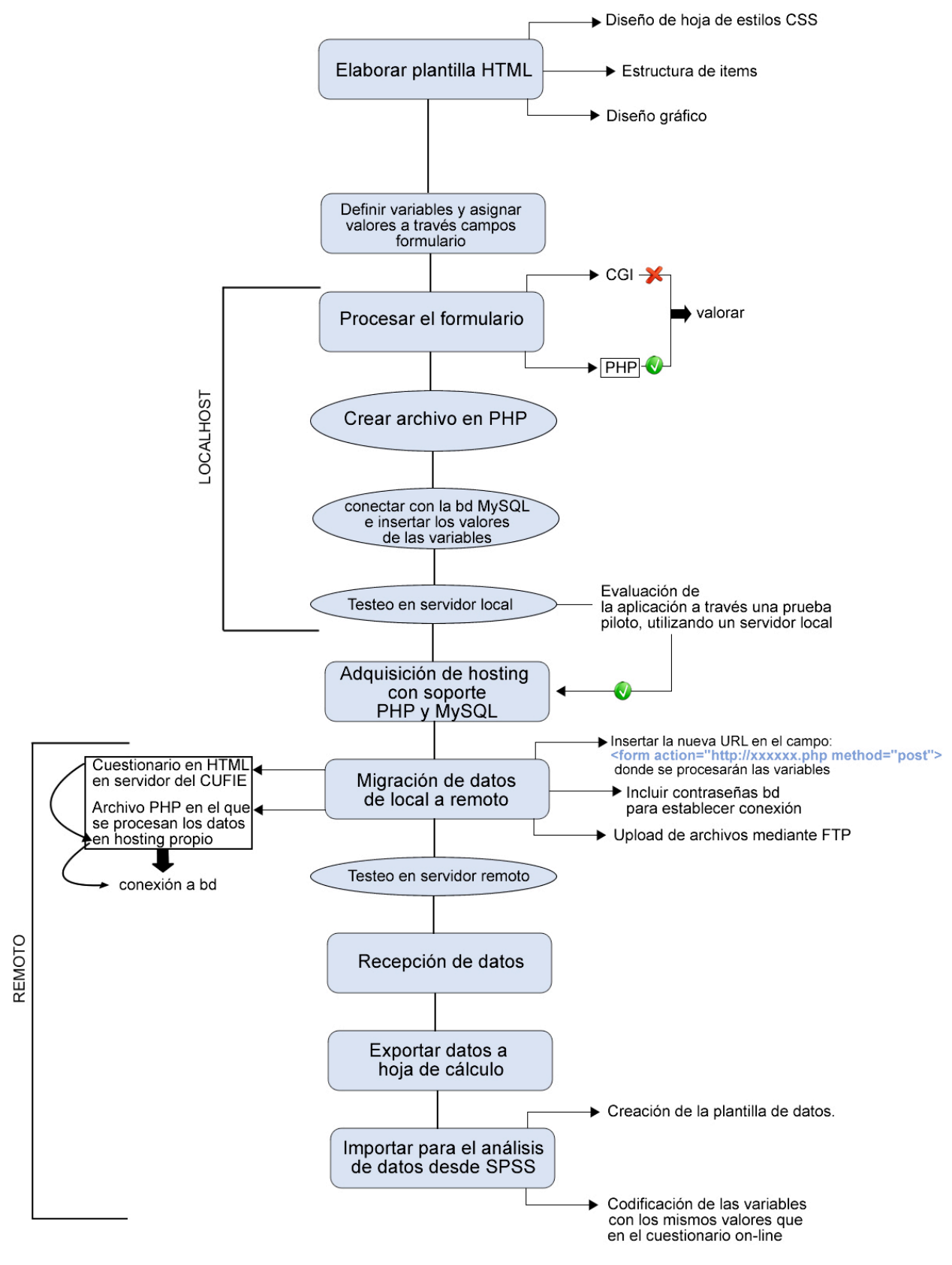

Figura 2: Fases desarrolladas en el diseño técnico del cuestionario on-line.

\section{RESULTADOS ALCANZADOS.}

Tal y como hacíamos constar en la introducción, en este artículo nos centraremos en dar a conocer los resultados obtenidos referentes al nivel de uso que el profesorado hace de herramientas y aplicaciones propias del área de programación y bases de datos bajo sistemas teleformativos.

Comenzaremos indicando que, los análisis desarrollados se articulan en dos tipos: análisis descriptivos y análisis inferenciales. 
Respecto a los primeros, cabe indicar que el hecho de emplear análisis descriptivos nos permitió (Gil, 2000; Vilà y Bisquerra, 2004; Vilà, 2006; Pérez López, 2005):

- Realizar una inspección de los datos, revisando y depurando posibles errores que contenga la matriz y que puedan alterar los resultados del estudio (Vilà y Bisquerra, 2004). Por ejemplo, valores perdidos o detección de outliers ${ }^{6}$.

- Situarnos en la realidad de los datos que poseemos, conociendo sus características y formándonos una idea lo más exacta posible de las mismas (Gil, 2000).

- Presentar, describir, organizar y resumir los datos observados, prestando especial atención a tres aspectos básicos como son la tendencia central, la dispersión y la forma de distribución.

En cuanto a los análisis inferenciales, el procedimiento estadístico que se utilizó, recurre a comparar las medias de las distribuciones de la variable cuantitativa en los diferentes grupos establecidos por la variable categórica. Si ésta tiene sólo dos categorías (dicotómica), la comparación de medias entre dos grupos independientes se lleva a cabo por el test $\mathbf{t}$ de Student; si tiene tres o más categorías, la comparación de medias entre tres o más grupos independientes se realiza a través de un modelo matemático más general, el análisis de la varianza (ANOVA). El problema es que en ambos casos, las pruebas estadísticas son exigentes con ciertos requisitos previos: la distribución normal de la variable cuantitativa en los grupos que se comparan y la homogeneidad de varianzas (homocedasticidad) en las poblaciones de las que proceden los grupos; su no cumplimiento conlleva necesariamente la necesidad de que recurramos a pruebas estadísticas no paramétricas. En este supuesto se optó por emplear la prueba $\mathbf{U}$ Mann Whitney para dos muestras independientes y Kruskal-Wallis para $k$ muestras independientes.

Una vez establecidas estas consideraciones, pasamos propiamente a exponer los resultados obtenidos en la investigación (área de programación y bases de datos). Comenzaremos para ello mostrando los datos de carácter descriptivo (ver tabla 2 y gráfico 1).

\footnotetext{
${ }^{6}$ sujetos con puntuaciones que se distancian notablemente de los modelos establecidos para el resto de individuos de la muestra.
} 


\begin{tabular}{|c|c|c|c|c|c|c|c|c|c|c|c|c|c|c|}
\hline \multicolumn{15}{|c|}{ COMPETENCIAS TÉCNICAS EN PROC } \\
\hline \multicolumn{15}{|c|}{ Nivel de aplicación/uso docente. } \\
\hline & \multicolumn{2}{|c|}{ NS/NC } & \multicolumn{2}{|c|}{ Muy bajo } & \multicolumn{2}{|c|}{ Bajo } & \multicolumn{2}{|c|}{ Medio } & \multicolumn{2}{|c|}{ Alto } & \multicolumn{2}{|c|}{$\begin{array}{l}\text { Muy } \\
\text { Alto }\end{array}$} & \multirow{2}{*}{ Media } & \multirow{2}{*}{ DT } \\
\hline & $n$ & $\%$ & $n$ & $\%$ & $n$ & $\%$ & $n$ & $\%$ & $n$ & $\%$ & $\mathrm{n}$ & $\%$ & & \\
\hline $\begin{array}{r}\text { Lenguajes de } \\
\text { marcado } \\
\text { (i10.12) }\end{array}$ & 15 & 9,0 & 105 & 63,3 & 21 & 12,7 & 11 & 6,6 & 7 & 4,2 & 7 & 4,2 & 1,46 & 1,153 \\
\hline $\begin{array}{r}\text { Lenguajes de } \\
\text { servidor para } \\
\text { diseño de } \\
\text { webs (i10.13) }\end{array}$ & 16 & 9,6 & 108 & 65,1 & 12 & 7,2 & 16 & 9,6 & 5 & 3,0 & 9 & 5,4 & 1,48 & 1,215 \\
\hline $\begin{array}{r}\text { Sistemas de } \\
\text { bases de } \\
\text { datos (i10.14) }\end{array}$ & 14 & 8,4 & 109 & 65,7 & 18 & 10,8 & 12 & 7,2 & 6 & 3,6 & 7 & 4,2 & 1,45 & 1,136 \\
\hline $\begin{array}{r}\text { Aplicaciones } \\
\text { interactivas } \\
\text { web } 2.0 \\
\text { (i10.15) }\end{array}$ & 23 & 13,9 & 111 & 66,9 & 13 & 7,8 & 13 & 7,8 & 3 & 1,8 & 3 & 1,8 & 1,22 & 968 \\
\hline
\end{tabular}

Tabla 2.- Extracto de los ítems pertenecientes al bloque II del cuestionario sobre formación y aplicación de sistemas de e-learning: Área de "nivel de aplicación/uso docente de herramientas de programación y bases de datos bajo sistemas de e-learning".

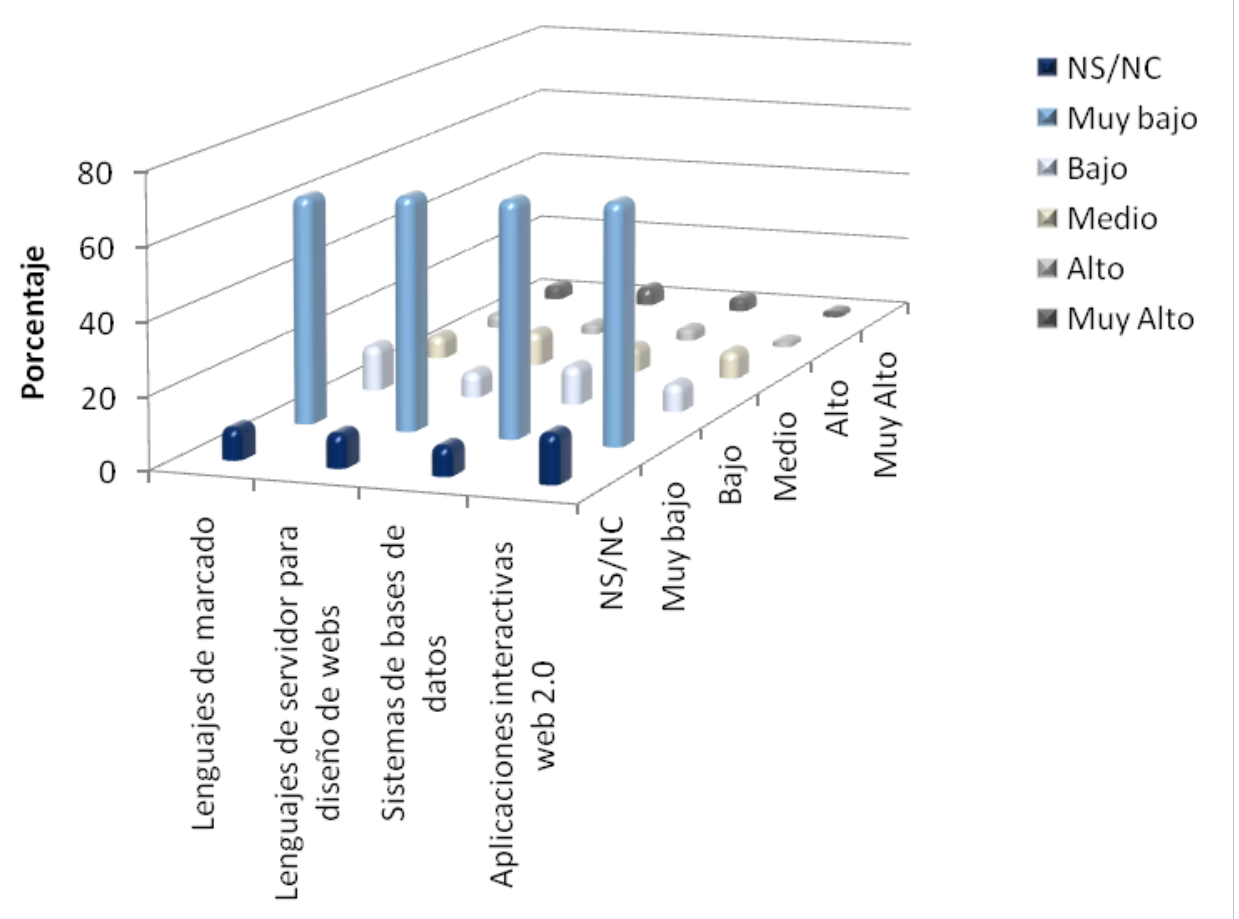

Gráfico 1: Histograma con porcentajes de la categoría "nivel de aplicación/uso" en herramientas y aplicaciones del área de programación y bases de datos. 
Tal y como se muestra en la tabla 2 y en el gráfico 1, las puntuaciones obtenidas son muy bajas, no en vano las tasas de respuesta se concentran en la categoría de uso "muy bajo".

Así, los "lenguajes de marcado", obtienen un porcentaje de respuesta en la categoría "muy bajo" de $63,3 \%$ y de $12,7 \%$ en la de "bajo". Por su parte, el uso que el profesorado hace de los "lenguajes de servidor para diseño de webs" es de un 65,1\% en la categoría de "muy bajo" y de $7,2 \%$ en la de "bajo". En lo que atañe a los "sistemas de bases de datos", la gran mayoría del profesorado (un 65,7\%) manifiesta tener un nivel de uso "muy bajo". Lo mismo sucede con las "aplicaciones interactivas web 2.0 ", donde un 66,9\% señala que posee un nivel de aplicación de las mismas "muy bajo".

Centrándonos en los análisis de tipo inferencial, es preciso indicar en este punto que a través de los mismos pretendíamos conocer el grado de asociación o independencia entre las variables categóricas e independientes: "sexo", "edad", "experiencia docente", "experiencia docente universitaria utilizando entornos virtuales", "categoría administrativa" y "ámbito científico" (variables todas ellas pertenecientes al bloque I del cuestionario: "datos de identificación" de la muestra), respecto a las competencias técnicas en el uso y aplicación de herramientas de programación y bases de datos manifestadas por los docentes (que actúan como variables dependientes en el modelo).

Así pues, las preguntas a las que pretendíamos dar respuesta en el análisis de relaciones entre las citadas variables, eran las siguientes (ver tabla 3 ):

\begin{tabular}{|c|c|c|}
\hline \multirow[b]{2}{*}{ VARIABLES } & DEPENDIENTES $\rightarrow$ & \multirow[b]{2}{*}{$\begin{array}{l}\text { Nivel de aplicación/uso herramientas programación } \\
\qquad y \text { bases de datos }\end{array}$} \\
\hline & INDEPENDIENTES $\downarrow$ & \\
\hline & Sexo & $\begin{array}{l}\text { ¿Hay diferencias en el uso docente de aplicaciones } \\
\text { del área de programación y bases de datos según los } \\
\text { individuos sean profesores o profesoras? }\end{array}$ \\
\hline & Edad & $\begin{array}{c}\text { ¿Hay diferencias en el uso docente de sistemas de } \\
\text { programación y bases de datos según la edad de los } \\
\text { docentes? }\end{array}$ \\
\hline \multicolumn{2}{|c|}{ Experiencia docente } & $\begin{array}{l}\text { ¿Está relacionado el uso que el profesorado hace de } \\
\text { herramientas del área de programación y bases de } \\
\text { datos con la experiencia docente? }\end{array}$ \\
\hline \multicolumn{2}{|c|}{ Experiencia docente EV } & $\begin{array}{c}\text { ¿Existen diferencias en el uso docente de sistemas } \\
\text { de programación y bases de datos según la } \\
\text { experiencia docente universitaria utilizando } \\
\text { entornos virtuales? }\end{array}$ \\
\hline \multicolumn{2}{|c|}{ Categoría administrativa } & $\begin{array}{l}\text { ¿Hay diferencias en el uso docente de sistemas de } \\
\text { programación y bases de datos según la categoría } \\
\text { administrativa del profesorado? }\end{array}$ \\
\hline \multicolumn{2}{|c|}{ Ámbito científico } & $\begin{array}{l}\text { ¿Está relacionado el uso que el profesorado hace de } \\
\text { herramientas del área de programación y bases de } \\
\text { datos según el ámbito científico al que pertenece el }\end{array}$ \\
\hline
\end{tabular}




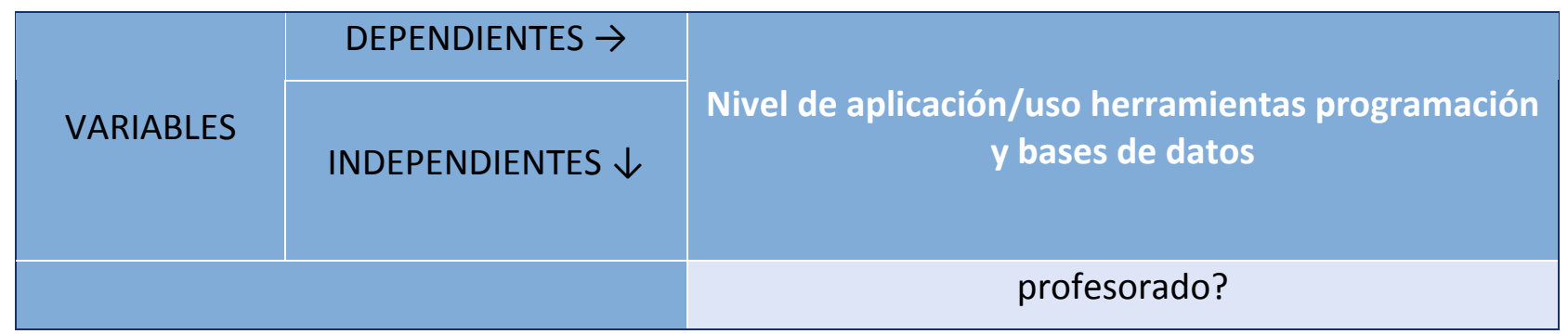

Tabla 3: Preguntas clave para conocer el grado de asociación o independencia que presetan las variables categóricas personales y profesionales del profesorado de la UDC respecto al área de "programación y bd".

Para dar respuesta a las preguntas formuladas en la tabla 3, se realizaron las correspondientes pruebas de normalidad con el fin de determinar la idoneidad de aplicar una prueba paramétrica (en caso de que las variables sigan una distribución normal) o bien no paramétrica (si las variables no se distribuyen siguiendo una Ley Normal).

En esta ocasión, todos los análisis efectuados a través de los estadísticos Kolmogorov-Smirnov y Shapiro-Wilk (tabla 4), demuestran que las diversas variables a contrastar no siguen un criterio de normalidad, obteniéndose un p-valor inferior a 0,05 en todos los grupos, lo que nos obligó a desarrollar pruebas no paramétricas (Mann Whitney para variables dicotómicas y KruskalWallis para variables politómicas con $\mathrm{K}$ muestras independientes). La tabla 4 muestra en conjunto, todas las pruebas de normalidad realizadas para las diversas variables objeto de estudio.

\begin{tabular}{|c|c|c|c|c|c|c|c|c|}
\hline \multicolumn{9}{|c|}{ Factor de exploración: sexo. } \\
\hline & & \multirow{2}{*}{\multicolumn{2}{|c|}{ sexo }} & \multicolumn{5}{|c|}{ Kolmogorov-Smirnov(a) } \\
\hline & & & & \multicolumn{2}{|c|}{ Estadístico } & \multicolumn{2}{|l|}{ gl } & Sig. \\
\hline \multirow{2}{*}{\multicolumn{2}{|c|}{ uso programación y bd }} & \multicolumn{2}{|c|}{ profesor } & \multicolumn{2}{|c|}{162} & \multicolumn{2}{|c|}{113} & ,000 \\
\hline & & \multicolumn{2}{|c|}{ profesora } & \multicolumn{2}{|c|}{ 194 } & \multicolumn{2}{|r|}{53} & 000 \\
\hline \multicolumn{9}{|c|}{ Factor de exploración: edad. } \\
\hline & \multirow[t]{2}{*}{ edad } & \multicolumn{4}{|c|}{ Kolmogorov-Smirnov(a) } & \multicolumn{3}{|c|}{ Shapiro-Wilk } \\
\hline & & & Estadístico & gl & Sig. & Estadístico & gl & Sig. \\
\hline \multirow[t]{4}{*}{$\begin{array}{l}\text { uso programación } \\
\text { y bd }\end{array}$} & $\begin{array}{l}\text { de } 25 \text { a } \\
35\end{array}$ & & ,184 & 32 & ,008 & ,864 & 32 & 001 \\
\hline & $\begin{array}{l}\text { de } 36 \text { a } \\
45\end{array}$ & & ,156 & 77 & ,000 & 919 & 77 & 000 \\
\hline & $\begin{array}{l}\text { de } 46 \text { a } \\
55\end{array}$ & & 177 & 39 & ,003 & 941 & 39 & 040 \\
\hline & $\begin{array}{l}\text { de } 56 \text { a } \\
65\end{array}$ & & ,255 & 17 & ,004 & ,786 & 17 & ,001 \\
\hline \multicolumn{9}{|c|}{ Factor de exploración: experiencia docente. } \\
\hline & \multicolumn{2}{|c|}{ Experiencia } & \multicolumn{3}{|c|}{ Kolmogorov-Smirnov(a) } & \multicolumn{3}{|c|}{ Shapiro-Wilk } \\
\hline
\end{tabular}






a Corrección de la significación de Lilliefors 
Tabla 4: Pruebas de normalidad (factores de exploración: sexo, edad, experiencia docente, experiencia docente utilizando EV, categoría administrativa y ámbito científico).

Centrándonos en la variable "sexo", y según los datos obtenidos en la prueba Mann-Whitney (tabla 5), podemos decir que no existen diferencias significativas en el nivel de uso en programación y bases de datos según el sexo del profesorado, ya que los niveles de significación obtenidos no son significativos al nivel alfa de 0,05. Es decir, no existe relación significativa entre las variables aplicación/uso en programación y sistemas gestores de bases de datos respecto al sexo.

\begin{tabular}{|c|c|c|c|c|c|c|}
\hline $\begin{array}{l}\text { Variables a } \\
\text { contrastar }\end{array}$ & sexo & $\mathrm{N}$ & $\begin{array}{c}\text { Rango } \\
\text { promedio }\end{array}$ & $\begin{array}{c}\text { Suma de } \\
\text { rangos }\end{array}$ & \multicolumn{2}{|c|}{ Estadísticos de contraste } \\
\hline \multirow{3}{*}{$\begin{array}{l}\text { uso programación } \\
\text { y bd }\end{array}$} & profesor & 113 & 83,83 & 9473,00 & \multirow{3}{*}{$\begin{array}{l}\text { U de Mann-Whitney } \\
\text { W de Wilcoxon } \\
\text { Z } \\
\text { Sig. asintót. } \\
\text { (bilateral) }\end{array}$} & \multirow{3}{*}{$\begin{array}{r}2957,000 \\
4388,000 \\
-, 131 \\
, 896\end{array}$} \\
\hline & profesora & 53 & 82,79 & 4388,00 & & \\
\hline & Total & 166 & & & & \\
\hline
\end{tabular}

Tabla 5: Prueba Mann-Whitney (variable de agrupación "sexo")

En lo que atañe a la variable "edad", la prueba Kruskal-Wallis (tabla 6) nos revela que debemos aceptar la hipótesis nula de independencia, de forma que podemos concluir que el nivel de aplicación/uso de herramientas del área de programación y bases de datos, no está relacionado con la edad del profesorado, ya que el p-valor obtenido de 0,084 es superior al nivel alfa de 0,05 . No obstante, si visualizamos los rangos promedio de la tabla 6 , se puede observar cómo son los profesores más jóvenes los que en mayor medida utilizan este tipo de herramientas. De hecho existe una diferencia bastante notable entre el intervalo de edad situado entre 25-35 años (con un rango promedio de 95,88), frente a los docentes situados en edades comprendidas entre los 56 y 65 años (con un rango promedio de 63,82).

\begin{tabular}{|c|l|r|r|r|r|}
\hline Variables a contrastar & edad & N & \multicolumn{1}{|c|}{$\begin{array}{c}\text { Rango } \\
\text { promedio }\end{array}$} & \multicolumn{2}{|c|}{ Estadísticos de contraste } \\
\hline uso programación y bd & de 25 a 35 & 32 & 95,88 & \\
& de 36 a 45 & 77 & 86,05 & Chi -cuadrado & 6,645 \\
& de 46 a 55 & 39 & 74,77 & gl & 3 \\
& de 56 a 65 & 17 & 63,82 & Sig. asintót. &, 084 \\
\hline & Total & 165 & & & \\
\hline
\end{tabular}

Tabla 6: Prueba de Kruskal-Wallis (variable de agrupación:"edad")

En cuanto a la variable "experiencia docente", cabe indicar que con un nivel alfa prefijado de 0,05 , la prueba Kruskal-Wallis (tabla 7) nos aporta valores no significativos para la variable "uso programación y bases de datos" ( $p$-valor=0,080), lo que se traduce en que no existen 
diferencias significativas entre el uso que el profesorado realiza con herramientas de programación y bases de datos respecto a la experiencia docente. Sin embargo, de una forma similar a lo que sucedía con la variable "edad", son los profesores noveles los que detentan mayores rangos promedio frente a aquellos docentes con mayor experiencia. En cierto sentido resulta lógico, puesto que un menor nivel de experiencia docente suele corresponderse también con edades jóvenes.

\begin{tabular}{|c|c|c|c|c|c|}
\hline $\begin{array}{l}\text { Variables a } \\
\text { contrastar }\end{array}$ & $\begin{array}{c}\text { Experiencia } \\
\text { docente }\end{array}$ & N & $\begin{array}{l}\text { Rango } \\
\text { promedio }\end{array}$ & \multicolumn{2}{|c|}{ Estadísticos de contraste } \\
\hline \multirow{6}{*}{$\begin{array}{l}\text { uso programación y } \\
\text { bd }\end{array}$} & menos de 3 años & 12 & 99,79 & \multirow{6}{*}{$\begin{array}{l}\text { Chi -cuadrado } \\
\text { gl } \\
\text { Sig. asintót. }\end{array}$} & \multirow{6}{*}{$\begin{array}{r}8,342 \\
4 \\
, 080\end{array}$} \\
\hline & entre 4 y 6 años & 15 & 93,73 & & \\
\hline & entre 7 y 18 años & 95 & 83,67 & & \\
\hline & entre 19 y 30 años & 30 & 62,13 & & \\
\hline & entre 31 y 40 años & 10 & 78,70 & & \\
\hline & Total & 162 & & & \\
\hline
\end{tabular}

Tabla 7: Prueba de Kruskal-Wallis (variable de agrupación:"experiencia docente")

En lo que respecta a la variable "experiencia docente universitaria utilizando entornos virtuales", la prueba Kruskal-Wallis (tabla 8) indica que existen diferencias significativas en el nivel de uso en programación y bases de datos ( $p$-valor=0,006), según la experiencia docente universitaria que posee el profesorado en entornos virtuales. De hecho, si analizamos los rangos promedio podemos comprobar cómo a mayor experiencia docente universitaria utilizando entornos virtuales, existen mayores niveles de uso. Verbigracia, los profesores con una experiencia entre 5 y 6 años, obtienen un rango promedio de 95,71 frente al 61,2 del profesorado con menos de un año de experiencia, y al 65,09 obtenido por los docentes con un nivel de experiencia entre uno y dos años.

\begin{tabular}{|c|c|c|c|c|c|}
\hline $\begin{array}{l}\text { Variables a } \\
\text { contrastar }\end{array}$ & $\begin{array}{c}\text { Experiencia } \\
\text { docente } \\
\text { universitaria } \\
\text { utilizando } \\
\text { entornos virtuales }\end{array}$ & $\mathbf{N}$ & $\begin{array}{l}\text { Rango } \\
\text { promedio }\end{array}$ & \multicolumn{2}{|c|}{ Estadísticos de contraste } \\
\hline \multirow{6}{*}{$\begin{array}{l}\text { uso programación y } \\
\text { bd }\end{array}$} & menos de 1 año & 26 & 61,62 & \multirow{6}{*}{$\begin{array}{l}\text { Chi -cuadrado } \\
\text { gl } \\
\text { Sig. asintót. }\end{array}$} & \multirow{6}{*}{$\begin{array}{r}14,281 \\
4 \\
, 006\end{array}$} \\
\hline & entre 1 y 2 años & 37 & 65,09 & & \\
\hline & entre 3 y 4 años & 55 & 91,31 & & \\
\hline & entre 5 y 6 años & 17 & 95,71 & & \\
\hline & más de 6 años & 25 & 88,82 & & \\
\hline & Total & 160 & & & \\
\hline
\end{tabular}

Tabla 8: Prueba de Kruskal-Wallis (variable de agrupación:"experiencia docente universitaria utilizando entornos virtuales"). 
Respeto a la variable "uso programación y bd", el p-valor obtenido de 0,056 (ver tabla 9), señala que se encuentra en los límites de la no significación, aunque observando las diferencias de los rangos promedio entre funcionarios y contratados, optaremos por considerar que existe relación entre el nivel de aplicación de herramientas de programación y bases de datos respecto a la categoría administrativa del profesorado. En este sentido, es el profesorado contratado el que obtiene unos niveles de aplicación y uso de herramientas de programación y bases de datos más elevadas que el profesorado funcionario. No obstante, reiteramos que el pvalor se halla en los límites de la no significación.

\begin{tabular}{|l|l|r|r|r|r|r|}
\hline $\begin{array}{l}\text { Variables a } \\
\text { contrastar }\end{array}$ & $\begin{array}{c}\text { Categoría } \\
\text { administrativa }\end{array}$ & N & $\begin{array}{c}\text { Rango } \\
\text { promedio }\end{array}$ & $\begin{array}{c}\text { Suma } \\
\text { de } \\
\text { rangos }\end{array}$ & \multicolumn{2}{|c|}{ Estadísticos de contraste } \\
\hline $\begin{array}{l}\text { uso programación } \\
\text { y bd }\end{array}$ & funcionario & 105 & 77,22 & 8108,50 & U de Mann-Whitney & 2543,500 \\
& contratado & 59 & 91,89 & 5421,50 & W de Wilcoxon & 8108,500 \\
& Total & 164 & & & Z & $-1,912$ \\
& & & & & Sig. asintót. \\
(bilateral) &, 056 \\
\hline
\end{tabular}

Tabla 9: Prueba Mann-Whitney (variable de agrupación "categoría administrativa")

Finalmente, podemos decir que la prueba U Mann Whitney (tabla 10), indica la no existencia de diferencias significativas entre la variable "uso programación y bd" ( $p$-valor=0,164) respecto al ámbito científico del que proviene el profesorado, el cual hemos dicotomizado en: "científicosanitario/técnicas" y "humanas/jurídico-sociales". No obstante, es preciso señalar que los rangos promedio señalan que es el ámbito científico-sanitario/técnicas con un 87,48 el que utiliza en mayor medida herramientas y aplicaciones relacionadas con la programación y bases de datos en sistemas teleformativos.

\begin{tabular}{|c|c|c|c|c|c|c|}
\hline $\begin{array}{l}\text { Variables a } \\
\text { contrastar }\end{array}$ & $\begin{array}{c}\text { Categoría } \\
\text { administrativa }\end{array}$ & N & $\begin{array}{c}\text { Rango } \\
\text { promedio }\end{array}$ & $\begin{array}{c}\text { Suma } \\
\text { de } \\
\text { rangos }\end{array}$ & \multicolumn{2}{|c|}{ Estadísticos de contraste } \\
\hline \multirow[t]{3}{*}{$\begin{array}{l}\text { uso programación } \\
\text { y bd }\end{array}$} & $\begin{array}{l}\text { cientifico- } \\
\text { sanitario y } \\
\text { tecnicas }\end{array}$ & 104 & 87,48 & 9097,50 & \multirow{3}{*}{$\begin{array}{l}\text { U de Mann-Whitney } \\
\text { W de Wilcoxon } \\
\text { Z } \\
\text { Sig. asintót. } \\
\text { (bilateral) }\end{array}$} & \multirow{3}{*}{$\begin{array}{r}2810,500 \\
4763,500 \\
-1,392 \\
, 164\end{array}$} \\
\hline & $\begin{array}{l}\text { Humanas y } \\
\text { jurídico- } \\
\text { sociales }\end{array}$ & 62 & 76,83 & 4763,50 & & \\
\hline & Total & 166 & & & & \\
\hline
\end{tabular}

Tabla 10: Prueba Mann-Whitney (variable de agrupación "ámbito científico")

\section{DISCUSIÓN DE LOS RESULTADOS.}


Los resultados obtenidos en el estudio nos llevan a señalar desde una óptica global que el nivel de aplicación y uso presentado por los docentes en el área de programación y bases de datos son muy discretos, ya que las puntuaciones medias alcanzadas reflejan unos índices bajos, siendo los "lenguajes de marcado y los lenguajes de servidor para el diseño de webs los que mayor puntuación media han obtenido (1,46 y 1,48, respectivamente).

Por otra parte, resulta interesante señalar que en base al ámbito científico, son los docentes que imparten asignaturas de corte técnico los que alcanzan mayores cuotas de formación. En buena medida este hecho se debe a que en las titulaciones de la rama técnica, como por ejemplo las ingenierías, se suelen emplear programas y aplicaciones muy específicas en las que es preciso que el profesorado posea competencias técnicas concretas.

Bajo un punto de vista inferencial, los contrastes estadísticos realizados indican la existencia de variables de carácter profesional que influyen significativamente en los niveles de aplicación y uso que manifiesta poseer el profesorado de la Universidad de A Coruña en el área de programación y bases de datos bajo sistemas e-learning. En concreto, se han encontrado diferencias significativas en base a la "experiencia docente utilizando entornos virtuales" y a la "categoría administrativa". Respecto a la primera, es preciso indicar que a mayor experiencia docente universitaria utilizando entornos virtuales, existen mayores niveles de uso. De esta forma, los profesores con una experiencia entre 5 y 6 años, obtienen un rango promedio de 95,71 frente al 61,2 del profesorado con menos de un año de experiencia, y al 65,09 obtenido por los docentes con un nivel de experiencia entre uno y dos años.

En cuanto a la categoría administrativa, es el profesorado contratado el que obtiene unos niveles de aplicación y uso de herramientas de programación y bases de datos más elevadas que el profesorado funcionario. No obstante, es preciso indicar que el p-valor obtenido se halla en los límites de la no significación. En otras investigaciones a nivel nacional como la desarrollada por Alba (2004) también se pone de manifiesto que, en líneas generales, el profesorado contratado suele poseer un nivel de formación y un grado de uso de herramientas de tele-enseñanza más elevado que el profesorado funcionario.

Por último, cabe señalar que los resultados alcanzados nos han aportado información relevante para que, desde un punto de vista institucional, se puedan implementar las acciones formativas necesarias que mejoren las competencias tecnológicas de los docentes en aquellas áreas que se han constatado como deficitarias.

\section{BIBLIOGRAFIA}

Alba, C. (2004). Estudio sobre la viabilidad de las propuestas metodológicas derivadas de la aplicación del crédito europeo por parte del profesorado de las universidades españoles, vinculadas a la utilización de las TICS en la docencia y la investigación, en Programa de Estudios y Análisis de la Secretaría de Estado de Educación y Universidades (EA2004-0042), http://www.mec.es/univ/html/informes/estudios_analisis/resultados_2004/ea0042/EA-20040042-ALBA-2-InformeGlobal.pdf (10/07/2009)

Area, M. (dir.) (2001). La oferta de educación superior a través de Internet. Análisis de los campus virtuales de las universidades españolas. En Programa de Estudios y Análisis de la Secretaría de Estado de Educación y Universidades (EA-E-A-7224). Recuperado el 23 de septiembre de 2007 de http://www.edulab.ull.es/campusvirtuales/informe/documentos.htm 
Ardizzone, P. y Rivoltella, P. C. (2004). E-learning. Métodos e instrumentos para la innovación de la enseñanza universitaria. Málaga: Ediciones Aljibe.

Arnal, J. del Rincón, D. y Latorre, A. (1992). Investigación educativa. Fundamentos y metodología. Barcelona: Labor.

Barro, S. (dir.). (2004). Las Tecnologías de la Información y de las Comunicaciones en el sistema universitario español. Madrid: CRUE.

Barro, S. y Burillo, P. (dir.). (2006). Las TIC en el sistema universitario español: un análisis estratégico. Madrid: CRUE.

Best, J. W. (1982). Cómo investigar en educación. Madrid: Morata.

Bisquerra, R. (coord.) (2004). Metodología de la investigación educativa. Madrid: Editorial la Muralla.

Buendía, L., Colás, P. y Hernández, R. (1998). Métodos de investigación en psicopedagogía. Madrid: McGraw-Hill.

Cabero, J. (dir.) (2002). Las TICs en la Universidad. Sevilla: Editorial MAD.

Cohen, L. y Manion, L. (1990). Métodos de investigación educativa. Madrid: La Muralla.

Fox, D. (1981). El proceso de investigación en educación. Pamplona, Eunsa.

Gil, J. A. (2000). Estadística e informática (SPSS) en la investigación descriptiva e inferencial. Madrid: UNED.

González Sanmamed, M. (1993). Perspectivas para el desarrollo del conocimiento profesional de los profesores en formación. Universidad de Santiago de Compostela. Tomo II. Tesis doctoral inédita.

González Sanmamed, M. (2004). La integración de las TIC en la UDC: el proyecto ITEM. En Sangrà, A. y González Sanmamed, M. La transformación de las universidades a través de las TIC: discursos y prácticas, pp. 121-125. Barcelona: UOC.

González Sanmmed, M. (2005). La integración de las Tecnologías de la Información y la Comunicación en la educación superior: experiencias en la UDC. En Raposo, M. y Sarceda, M. C. (coords.) Experiencias y prácticas educativas con nuevas tecnologías, pp. 69-87. Ourense: a.i.c.a. ediciones.

Hanna, D. E. (2002). La enseñanza universitaria en la era digital. Bacelona: Octaedro.

Infante, A. (2004). La enseñanza virtual en España ante el nuevo Espacio Europeo de Educación Superior. En Programa de Estudios y Análisis de la Secretaría de Estado de Educación y Universidades (EA.2004-0090). http://www.micinn.es/univ/proyectos2004/EA2004-0090.pdf (18/07/2009) 
Manzano, V. y Braña, T. (2005). Análisis de datos y técnicas de muestreo. En Lévy Mangin, J. P. y Varela, J. Análisis multivariable para las ciencias sociales, pp. 91-143. Madrid: Pearson Prentice Hall.

Marcelo, C. (2006). Las nuevas competencias en e-learning: ¿qué formación necesitan los profesionales del e-learning?. En Marcelo, C. (coord.). Prácticas de e-learning, pp. 22-45. Churriana de la Vega (Granada): Ediciones Octaedro.

McMillan, J. H. y Schumacher, S. (2005). Investigación educativa. Madrid: Pearson Addison Wesley.

Muñoz Carril, P. C. y González Sanmamed, M. (2009). Plataformas de teleformación y herramientas telemáticas. Barcelona: Editorial UOC.

Pérez López, C. (2005). Técnicas estadísticas con SPSS 12. Aplicaciones del análisis de datos. Madrid: Pearson Prentice Hall.

Prat, R. y Doval, E. (2005). Construcción y análisis de escalas. En Lévy Mangin, J. P. y Varela, J. Análisis multivariable para las ciencias sociales, pp. 43-89. Madrid: Pearson Prentice Hall.

Prendes, M. P. (dir.) (2009). Plataformas de campus virtual con herramientas de software libre: Análisis comparativo de la situación actual en las universidades españolas". En Programa de Estudios y Análisis, Secretaría de estado de Universidades e Investigación. http://www.um.es/campusvirtuales/informe_final_CVSL_SF.pdf (27/08/2009)

Sabariego, M. (2004). El proceso de investigación (parte 2). En Bisquerra, R. (coord.) Metodología de la investigación educativa, pp. 127-163. Madrid: Editorial la Muralla.

Salinas, J. (1999). "Enseñanza flexible, aprendizaje abierto. Las redes como herramientas para la formación". En EDUTEC, Revista Electrónica de Tecnología Educativa, № 10. http://www.uib.es/depart/gte/revelec10.html (16/03/2010)

Salinas, J. (dir.) (2007). Modelos didácticos en campus virtuales universitarios: patrones metodológicos generados por los profesores en procesos de enseñanza-aprendizaje en entornos virtuales (Ea2007-0121). En Programa de Estudios y Análisis, Secretaría de estado de Universidades $e$ Investigación. http://82.223.160.188/mec/ayudas/repositorio/20080530122028EA2007-0121-memoria.pdf (06/09/2009)

Sangrà, A. (coord.) (2004). Análisis de la oferta formativa por medios electrónicos de 1r. y 2o ciclo en las universidades españolas: estudio de su contribución al proceso de convergencia europea En Programa de Estudios y Análisis, Secretaría de estado de Universidades $e$ Investigación.

Solanas, M. (1997). Métodos de recerca. Barcelona: UOC-Proa.

Torrado, M. (2004). Estudios de encuesta. En Bisquerra, R. (coord.) Metodología de la investigación educativa, pp. 231-257. Madrid: Editorial la Muralla. 
Vilà, R. y Bisquerra, R. (2004). El análisis cuantitativo de los datos. En Bisquerra, R. (coord.) Metodología de la investigación educativa. pp. 259-271. Madrid: Editorial la Muralla.

Para citar este artículo:

MUÑOZ, Pablo César; GONZÁLEZ, Mercedes (2010) « ESTUDIO CUANTITATIVO SOBRE EL USO DOCENTE DE HERRAMIENTAS TELEFORMATIVAS EN EL ÁMBITO DE LA PROGRAMACIÓN Y BASES DE DATOS» [artículo en línea]. EDUTEC, Revista Electrónica de Tecnología Educativa. Núm. 32 / Mayo 2010. [Fecha de consulta: dd/mm/aa]. http://edutec.rediris.es/revelec2/revelec32/ISSN $1135-9250$. 